\title{
A microstructural model on the link between change in pore size distribution and wetting induced deformation in a compacted silt
}

\author{
Francesca Casini $^{(1)}$, Jean Vaunat ${ }^{(1)}$, Enrique Romero ${ }^{(1)}$ \\ Departamento de Ingeniería del Terreno, Cartográfica y Geofísica, Universitat \\ Politècnica de Catalunya, Barcelona, Spain. Corresponding author e-mail: \\ francesca.casini@upc.edu
}

\begin{abstract}
The water retention curve expressed in terms of water content or degree of saturation and suction is hysteretic, stress path dependent (on void ratio) and it depends on pore network. To properly describe its evolving nature, a multimodal retention model is proposed by considering a linear superposition of two subcurves of the Van Genuchten type. The model is constructed based on PSDs obtained with Mercury Intrusion Porosimetry (MIP) data at specified initial state, and thus, representative of this initial pore network configuration. Based on the WRC model proposed above the evolution of parameter $\chi$ will be evaluated based on the microstructural evolution of the pore network. The model is validated based on a series of oedometer samples performed at constant water content and then saturated at different initial void ratio and the same water content.
\end{abstract}

\section{INTRODUCTION}

The concept of effective stress and its extension from saturated to unsaturated conditions is a subject of interest in the last decades. A simple model for predicting the deformation induced by wetting has been proposed by Casini (2012) considering the variable $\sigma_{v}^{\prime}=\sigma_{v}-u_{a}+\chi\left(u_{a}-u_{w}\right)$ (Bishop, 1959) with $\chi=S_{r}$. The objective was to predict the deformation induced by wetting using the saturated parameters, the water retention curve and one additional parameter. The model may capture the progressive degradation induced by loading and wetting by linking Normal Compression Line (NCL) by the parameter $\chi$ and the water retention curve by porosity. However, as pointed out by Romero \& Vaunat (2000), two main mechanisms generally govern the storage of water inside a soil: the first mechanism is mainly related to free water flow inside the macropores between aggregates/grain; the second one is related to water adsorption at the intra-aggregate level. While the second mechanism is virtually independent of the void ratio, the first one is coupled with the mechanical response of the soil (Vaunat et al., 2000).

A deeper understanding of how microstructural features affect the water storage mechanism of compacted soils is of particular in geotechnical engineering (e.g. Romero et al., 2011; Casini et al., 2012). A new equation to evaluate the parameter $\chi$ is proposed in this work based on pore size distribution (PSD) obtained via microstructural investigation. The material used in the tests is an aeolian silt extracted from a layer of alluvial soil located at Jossigny, East of Paris, France. The behaviour of the saturated and unsaturated material was studied by various authors (Vicol, 1990; Cui, 1993; 
Casini, 2008). The evolving nature of PSD along generalised stress paths (with varying void ratio and water content) is taken into account by updating the pore network configuration as the hydraulic paths develop. The simulated results are compared with controlled-suction oedometer tests in which different wetting and drying paths are carried out at different initial void ratios.

\section{WATER RETENTION MODEL}

In the literature, the WRC obtained for the different kind of soil are usually best fitted with original or modified Van Genuchten (1980) models without taking into account the heterogeneity of the pore systems. Here the heterogeneity of the pore system induced by the compaction process is taking into account using a multimodal retention model, defined by Durner (1994) as a linear superposition of subcurves of the Van Genuchten type with the following equation:

$$
E_{\mathrm{w}}=\frac{e_{\mathrm{w}}-e_{\text {wres }}}{e-e_{\text {wres }}}=\sum_{i=1}^{k} w_{\mathrm{i}}\left[\frac{1}{1+(\alpha \cdot s)^{n_{\mathrm{i}}}}\right]^{m \mathrm{i}}
$$

where $k$ is the number of sub-system that assembled together give the global pore size distribution, $w_{i}$ are weighting factors for each sub-curves subjects to $0<w_{\mathrm{i}}<1$ and $\sum w_{\mathrm{i}}=1$. For the parameters of the sub-curves $\left(\alpha_{\mathrm{i}}, n_{\mathrm{i}}, m_{\mathrm{i}}\right)$ must be imposed the condition $\alpha_{\mathrm{i}}>0, m_{\mathrm{i}}>0, n_{\mathrm{i}}>0$. Here is imposed the additional constraint: $n_{\mathrm{i}}=1 /\left(1-m_{\mathrm{i}}\right)$.

Each sub-curve in Equation 2 differentiated two times with respect to the suction $\mathrm{s}$ give the relation between the suction pressure at the inflection point and the parameters given by the following expression: $s_{\mathrm{pi}}=m_{\mathrm{i}}^{1-m_{\mathrm{i}}} / \alpha_{\mathrm{i}}$ where $s_{\mathrm{pi}}$ is the suction at the inflection point in the $E_{\mathrm{w}}-S$ plane. Obtained $\alpha_{\mathrm{i}}$ from Equation (3) and substituting in equation (2) the water storage mechanisms in an heterogeneous porous medium became:

$$
E_{\mathrm{w}}=\frac{e_{\mathrm{w}}-e_{\mathrm{wres}}}{e-e_{\text {wres }}}=\sum_{i=1}^{k} w_{\mathrm{i}}\left[1+m_{\mathrm{i}}\left(s / s_{\mathrm{pi}}\right)^{1 / 1-m_{\mathrm{i}}}\right]^{m_{\mathrm{i}}}
$$

The equation 4 is used for model the WRC data obtained by MIP. The experimental data are modelled with a bi-modal water retention model, obtained setting $k=2$ in the above equation.

The pore capillary density function (PCF) is defined as: $P C F=\Delta E_{\mathrm{w}} / \Delta \log (s)$ where $E_{\mathrm{w}}$ is the effective degree of saturation as defined above.

The model is calibrated best fitting the PCF obtained by experimental results as reported in Figure 1. The area below the peak of lower suction is defined as $\mathbf{w}_{1}$ (macro area below the PCF) while $\mathrm{w}_{2}$ (micro area) is given by $\mathrm{w}_{2}=1-\mathrm{w}_{1}$ because the area below the curve corresponds to the unity. The suction at inflection points are named respectively $\mathrm{s}_{\mathrm{pM}}$ and $\mathrm{s}_{\mathrm{pm}}$ as reported in Figure 1 (Casini et al 2012).

The parameters obtained by the multimodal model have been calibrated as a function of void ratio and gravimetric water content based on twenty Mercury Intrusion Porosimetry (MIP) performed at five different void ratios e (0.5-0.9) and four gravimetric water content w (\%) (13-21\%) (see Casini et al 2012). 

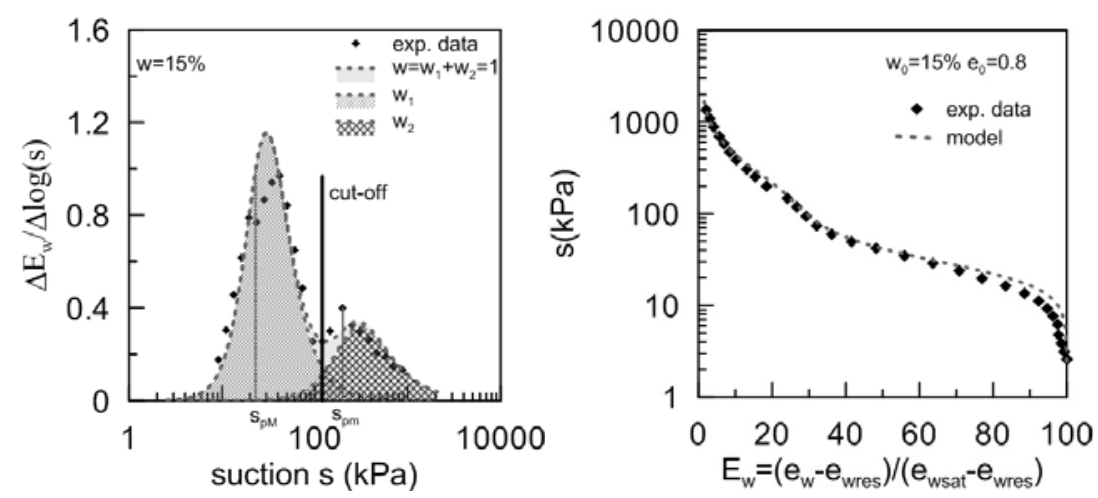

Figure 1. Laboratory versus model prediction: (a) Pore capillary function (PCF) macro micro; (b) WRC derived from PCF (Casini et al 2012)

\section{MODELLING FRAMEWORK}

The constitutive relationships for the mechanical behaviour are defined here in terms of Bishop's stress $\sigma_{i j}^{\prime}=\sigma_{i j}-u_{a} \delta_{i j}+\chi\left(u_{a}-u_{w}\right) \delta_{i j}$ and suction $s=u_{a}-u_{w}$, where $\sigma_{i j}$ is the total stress, $\chi$ is a weighting parameter, taking into account $S_{r}$ and $\delta_{i j}$ is the Kronecker delta.

In this work the weighting parameter $\chi$ is defined as:

$\chi=\frac{S_{r}-S_{\text {rmicro }}}{1-S_{\text {rmicro }}}$

where $S_{\text {rmicro }}$ is evalutated through the equation presented in the previous paragraph.

The vertical soil skeleton (effective) stress is defined as:

$\sigma_{v}^{\prime}=\sigma_{v}-u_{a}+\chi\left(u_{a}-u_{w}\right)$

Following the approach presented by Jommi (2000), the evolution of the preconsolidation vertical stress $\sigma_{v c}^{\prime}$ in unsaturated condition is defined as the product of a term that is function of the plastic volumetric strain $\left(\sigma_{v c s a t}^{\prime}\right)$ and a term depending on $\chi$ as reported below:

$\sigma_{v c}^{\prime}=\sigma_{v c s a t}^{\prime} e^{a(1-\chi)}$

where $a$ is a constant soil property that controls the rate of change in $\sigma_{v c}^{\prime}$ due to the variation of $\chi$. The hardening is a combination of a reversible component related to change in $\chi$ and an irreversible component dependent on the development of plastic strains.

The normal compression line under oedometer condition is given by: $e=B(\chi)-C_{c} \log \sigma_{v}^{\prime}$ with $B(\chi)=B+\left(C_{c}-C_{s}\right) a(1-\chi)$

where $\sigma_{v}^{\prime}$ is the vertical effective stress, $B(\chi)$ is the void ratio at $1 \mathrm{kPa}$ and $C_{c}$ is the compression index and $C_{\mathrm{s}}$ is the swelling index (for more details see Casini, 2012).

MODELLING EXPERIMENTAL RESULTS 
The model has been validated through three oedometer tests. The samples have been prepared by statically compacting at three different void ratios, then compressedunder a vertical loading $\sigma_{\mathrm{v}}=200 \mathrm{kPa}$ and finally saturated. The comparison is satisfactory in the two tests where the samples compress during saturation and in the case where the sample swells for the lower void ratio as shown in Figure 3.
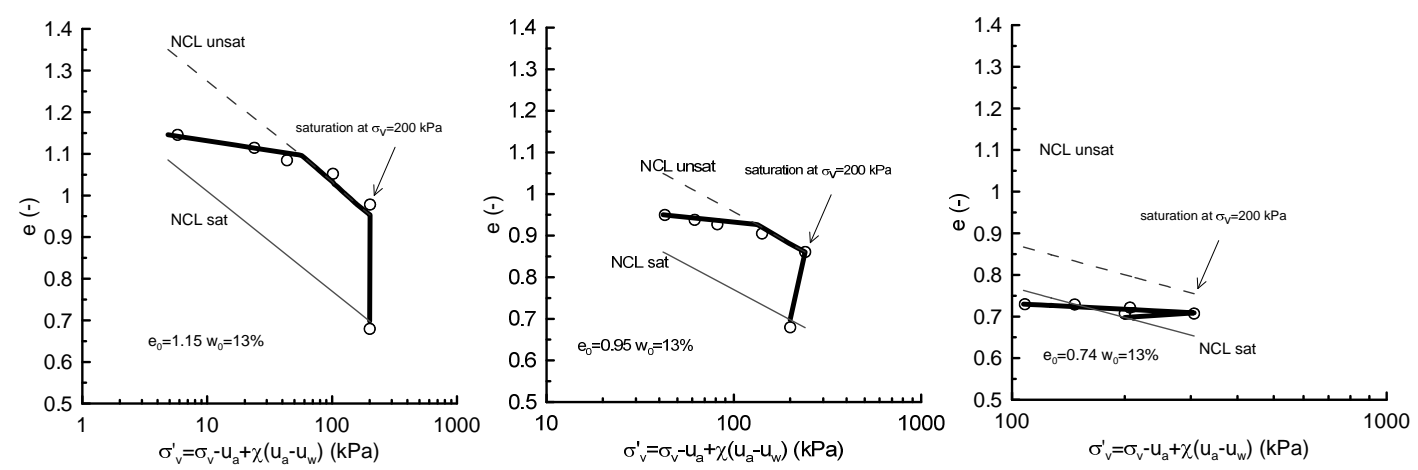

Figure 2 Comparison between model prediction and experimental results

\section{CONCLUSIONS}

A bi-modal water retention model has been proposed and calibrated with an extensive microstructural investigation performed on low plasticity silt. A simple expression has been used to model the Normal Compression Line of the unsaturated samples which depends on saturated parameters, the parameter $\chi$ and only one more parameter. The parameter $\chi$ depends on the macro effective degree of saturation, obtained by the total ones minus the micro degree of saturation evaluated through the water retention model. The comparison between model prediction and experimental results are satisfactory for samples compacted at the same water content and different void ratio.

\section{REFERENCES}

Bishop, A.W. (1959). The principle of effective stress. Tek. Ukebl. 106, No 39, 859863

Casini F (2008) Effetti del grado di saturazione sul comporta-mento meccanico di un limo, PhD Thesis, Universitá degli Studi di Roma "La Sapienza”, Italy.

Casini F. 2012. Deformation induced by wetting: a simple model. Canadian Geotechnical Journal 2012, 49(8): 954-960.

Casini F, Vaunat J., Romero E, Desideri A (2012) Consequences on water retention properties o fdouble-porosity features in a compacted silt. Acta Geotechnica 7: 139-150 DOI10.1007/s11440-012-0159-6.

Cui Y.J., (1993). “Etude du comportement d'un limon non saturé et de sa modélisation dans un cadre élasto-plastique” PhD Thesis, Ecole Nationale des Ponts et Chassées, Paris, France.

Durner W (1994) Hydraulic conductivity estimation for soils with heterogeneous pore structure. Water Resources Research 30(2): 211-223. 
Jommi C. (2000). Remarks on the constitutive modelling of unsaturated soils. Proc Int Workshop on Unsat Soils: Exp evid and Theor Approaches, Trento (Italy), Balkema:139-153.

Romero E, Della Vecchia G, Jommi C (2011) An insight into the water retention properties of compacted clayey soils. Géotechnique 61(4): 313-328

Romero E, Vaunat J (2000) Retention curves of deformable clays. Proc Int Workshop on Unsat Soils: Exp evid and Theor Approaches, Trento (Italy), Balkema: 91106

van Genuchten, MT (1980) A closed-form equation for pre-dicting the hydraulic conductivity of unsaturated soil. Soil Science Society American Journal, 44: 892-898.

Vaunat J, Romeo E, Jommi C. (2000) An elastoplastic hydro-mechanical model for unsaturated soils Proc Int Workshop on Unsat Soils: Exp evid and Theor Approaches, Trento (Italy), Balkema: 121-138

Vicol T (1990) Comportment hydraulique et mecanique d'un sol fin non sature application a la modelisation . PhD Thesis, Ecole Nationale des Ponts et Chassées, Paris, France. 